\title{
Characterization of mitochondrial NADH dehydrogenase $1 \alpha$ subcomplex 10 variants in cardiac muscles from normal Wistar rats and spontaneously hypertensive rats: Implications in the pathogenesis of hypertension
}

\author{
LIYING HUANG $^{1}$, XIAN JIN $^{1}$, LI XIA $^{2}$, XIAOLING WANG ${ }^{2}$, YUN YU $^{2}$, CUNFEI LIU $^{1}$, \\ DONGMIN SHAO $^{3}$, NINGYUAN FANG ${ }^{1}$ and $\mathrm{CHAO}_{\mathrm{MENG}}{ }^{1}$ \\ ${ }^{1}$ Department of Geriatrics, Ren Ji Hospital, School of Medicine, Shanghai Jiao Tong University, Shanghai 200001; \\ ${ }^{2}$ Department of Pathophysiology, School of Medicine, Shanghai Jiao Tong University, Shanghai 200025, P.R. China; \\ ${ }^{3}$ Section of Vascular Biology, National Heart and Lung Institute, \\ Imperial College London, London SW3 6LY, UK
}

Received January 11, 2015; Accepted November 5, 2015

DOI: $10.3892 / \mathrm{mmr} .2015 .4607$

\begin{abstract}
Mitochondrial dysfunction has been increasingly associated with the development of cardiovascular diseases, including hypertension and cardiac hypertrophy. In the present study, NADH dehydrogenase $1 \alpha$ subcomplex 10 (Ndufa10) was characterized from the left ventricular muscles of spontaneously hypertensive rats (SHRs) and normal Wistar Kyoto (WKY) rats. Western blot analysis demonstrated that there was a shift in the molecular weight (MW) and in the isoelectric point $(\mathrm{pI})$ of the Ndufa10 protein from SHRs and WKY rats. Mass spectrometric analysis revealed that the replacement of an aspartate residue with asparagine at amino acid position 120 was the biochemical difference between the two Ndufa10 isoforms. Further analysis using the bacterially expressed proteins Ndufa10-120N (WKY) and Ndufa10-120D (SHR) revealed that the shift in the $\mathrm{pI}$ and $\mathrm{MW}$ of the two Ndufa10 isoforms was solely caused by the amino acid mutation, and not by post-translational modifications. Since deficiencies of the mitochondrial complex I are the most common defects in the oxidative phosphorylation system, further studies are required to study the difference between the activities of the two Ndufa10 variants, and their role in the pathogenesis of hypertension.
\end{abstract}

Correspondence to: Dr Chao Meng or Dr Ningyuan Fang, Department of Geriatrics, Ren Ji Hospital, School of Medicine, Shanghai Jiao Tong University, 145 Middle Shandong Road, Shanghai 200001, P.R. China E-mail: chaomeng@shsmu.edu.cn

E-mail: fangny@sh163.net

Key words: NADH dehydrogenase $1 \alpha$ subcomplex 10, mutation, molecular weight, matrix-assisted laser desorption/ionization-time of flight-mass spectrometry, mobility

\section{Introduction}

As the most important subcellular organelle of energy metabolism in heart, mitochondria are also important in the balance of oxidative metabolism, $\mathrm{Ca}^{2+}$ homeostasis and for formation of reactive oxygen species (1). Abnormal mitochondrial respiration can result in oxidative stress via oxidative phosphorylation, and a compromised antioxidant system may lead to excessive oxidative stress in cardiovascular diseases, including hypertension, cardiac hypertrophy and atherosclerosis, indicating that mitochondria exert a fundamental role in disease progression (2-4).

Complex I (NADH:ubiquinone oxidoreductase) is the largest multimeric enzyme in the mitochondrial respiratory chain, which is responsible for electron transport and the generation of a proton gradient across the mitochondrial inner membrane to drive ATP production. It consists of 45 subunits with a combined mass of $\sim 980 \mathrm{kDa}$. Of those subunits, seven are encoded by mitochondrial DNA, whereas the other 38 subunits are encoded by nuclear genes. Ndufa10, a nuclear-encoded $42 \mathrm{kDa}$ protein, is located in the hydrophobic membrane $(5,6)$. A previous study in our laboratory reported that the NADH dehydrogenase $1 \alpha$ subcomplex 10 (Ndufa10) from the spontaneously hypertensive rat (SHR) strain exhibited a different profile from that of Wistar Kyoto (WKY) rats on two-dimensional (2D) fluorescence difference gel electrophoresis (7). The position of the Ndufa10 protein from the SHR strain demonstrated a horizontal shift to a lower isoelectric point (pI; $\sim 0.2 \mathrm{pH}$ units), with a slight increase in molecular weight (MW; $\sim 2 \mathrm{kDa}$ ) in the 2D gel compared with the protein from WKY. Genetic mutations and phosphorylation events are the most common causes for a pI shift decrease, and an MW increase, on performing 2D gel electrophoresis. Ndufa10 was reported to be phosphorylated by the cAMP-dependent protein kinase, and contains phosphothreonine residues in the steady state in bovine heart mitochondria (8-10). In addition to phosphorylation, the increase in MW could also be caused by 
other post-translational modifications, including deamidation, acetylation and methylation. At present, 33 modifications of a distinct chemical nature, targeting 59 specific residues of Ndufa10, have been found to be common to the acidic and basic forms (11). Previously, an A/G nucleotide transition at position 358 of Ndufa10, leading to an AAT-to-GAT mutation and, consequently, an Asn (N) to Asp (D) mutation at position 120 of the Ndufa10 amino acid sequence, was identified in our laboratory. However, the estimated difference in the MW caused by this mutation was only $\sim 1 \mathrm{Da}$, which did not match the apparent $\mathrm{MW}$, as determined from 2D gel electrophoretic and western blot analyses. It may be speculated that the mutation already co-existed, although with certain modifications. The aim of the present study was to determine the unique characteristics of the Ndufa10 protein from SHRs using 2D gel electrophoresis.

\section{Materials and methods}

$2 D$ gel electrophoresis. The isolation of the mitochondria from the left ventricles of 20-week-old WKY and SHR male rats was performed, as described previously (7). All the animals were obtained from the Shanghai SLAC Laboratory Animal Co., Ltd. (Shanghai, China) and housed in a temperature-controlled environment $\left(22-24^{\circ} \mathrm{C}\right)$ with a $12 / 12 \mathrm{~h}$ light-dark cycle with free access to food and water. All the procedures were approved by the local ethics committee of the School of Medicine, Shanghai Jiao Tong University (Shanghai, China; approval no. 2014069). Aliquots of $40 \mu \mathrm{g}$ protein from each sample were diluted in rehydration solution [8 M urea, 2\% (w/v) CHAPS, $65 \mathrm{mM}$ dithiothreitol (DTT) and 0.5\% (v/v) Bio-lyte 3/10 ampholyte; Bio-Rad Laboratories, Inc., Hercules, CA, USA)], adjusting the total volume to $125 \mu \mathrm{l}$ prior to applying the solution to non-linear IPG strips (7 cm in length; pH 3-10; Bio-Rad Laboratories, Inc.) using the following rehydration and running conditions: $200 \mathrm{~V}$ for $30 \mathrm{~min}, 500 \mathrm{~V}$ for $1 \mathrm{~h}, 1,000 \mathrm{~V}$ for $2 \mathrm{~h}$ and $4,000 \mathrm{~V}$ until $10,000 \mathrm{~V} / \mathrm{h}$ were reached. Following the completion of the isoelectric focusing procedure, the IPG strips were equilibrated for $30 \mathrm{~min}$ with $50 \mathrm{mM}$ Tris- $\mathrm{HCl}$ (pH 8.8), $6 \mathrm{M}$ urea, 30\% glycerol and 2\% SDS, followed by reduction with $1 \%$ DTT and alkylation with $2.5 \%$ iodoacetamide (Bio-Rad Laboratories, Inc.). The 2D separations were performed on $12 \%$ SDS-PAGE gels, and subsequently the proteins were transferred onto nitrocellulose membranes. The membranes were blocked with $5 \%$ nonfat dry milk solution for $1 \mathrm{~h}$ at room temperature. The membranes were blotted with mouse monoclonal anti- $\beta$-tubulin (dilution, 1:1,000; cat. no. T4026; Sigma-Aldrich, St. Louis, MO, USA) and chicken polyclonal anti-human Ndufa10 (dilution, 1:2,000; cat.no. ab19131; Abcam, Cambridge, UK) separately, followed by incubation with horseradish peroxidase-conjugated horse anti-mouse IgG (H+L) (dilution, 1:2,000; cat. no. 7076; Cell Signaling Technology, Inc., Danvers, MA, USA) and peroxidase-labeled goat anti-chicken $\operatorname{IgG}(\mathrm{H}+\mathrm{L})$ (dilution, 1:2,000; cat. no. 14-24-06; KPL, INC., Gaithersburg, MD, USA) . The membranes were developed using luminol-based enhanced chemiluminescence reagent (SuperSignal ${ }^{\mathrm{TM}}$ West Pico Chemiluminescent Substrate; Pierce Biotechnology, Inc., Rockford, IL, USA).
DNA sequencing. The genomic DNA was extracted from blood leukocytes from WKY and SHR rats aged 20 weeks using a blood genomic DNA extraction system [Tiangen Biotech (Beijing) Co., Ltd, Beijing, China], according to the manufacturer's protocol. Exon 3 of the Ndufa10 gene, where the mutation resides, was PCR-amplified and sequenced using, as the forward primer, 5'-TGGTTGTAGAGAACGGAGAAG CTC-3', and as the reverse primer, 5'-CTGCATCAACAGGGA TTTTGAGG-3'.

Plasmid construction and the transformation of prokaryotic cells. The total RNA was extracted from WKY and SHR heart tissue using Invitrogen TRIzol ${ }^{\circledR}$ reagent (Thermo Fisher Scientific, Waltham, MA, USA), according to the manufacturer's protocol. Samples of $1 \mu \mathrm{g}$ total RNA were used for cDNA synthesis using the Takara RNA polymerase chain reaction (PCR) kit (Takara Bio, Dalian, China), according to manufacturer's protocol. The cDNA encoding Ndufa10 was amplified by PCR using Pyrobest ${ }^{\mathrm{TM}}$ DNA polymerase and the Takara PCR kit (Takara Bio) in a final volume of $50 \mu 1$, containing the forward primer, 5'-CACCCATGGCCTTGA GGTTGCTG-3', and the reverse primer, 5'-CCACTCGAG TCACTTCAGCCAGATCCA-3'. The forward and reverse primers contained an NcoI site and an XhoI site, respectively. The thermal cycle for a hot-start PCR was: $95^{\circ} \mathrm{C}$ for $5 \mathrm{~min} ; 40$ cycles of $95^{\circ} \mathrm{C}$ for $30 \mathrm{sec} ; 65^{\circ} \mathrm{C}$ for $30 \mathrm{sec} ; 72^{\circ} \mathrm{C}$ for $1 \mathrm{~min} 30 \mathrm{sec}$; and, finally, $72^{\circ} \mathrm{C}$ for $10 \mathrm{~min}$. The amplified DNA fragments were separated using a $1 \%$ agarose gel. Subsequently, bands of the correct size $(1.068 \mathrm{~kb})$ were cut from the gel and recovered using the GenClean ${ }^{\mathrm{TM}}$ agarose gel DNA recovery kit I (Shanghai Generay Biotech Co., Ltd., Shanghai, China), prior to subcloning into Novagen pET28a-c (+) plasmids (EMD Millipore, Billerica, MA, USA) using the NcoI and XhoI sites. The resultant recombinant plasmids were subsequently transformed into DH5 $\alpha$ cells (Tiangen biotech (Beijing) Co., Ltd.). Following DNA sequencing to verify the constructs, the wild-type and mutant constructs were transformed into competent BL21(DE3) cells (Tiangen biotech (Beijing) Co., Ltd.) for recombinant protein production. Single colonies were inoculated into Luria-Bertani (LB) media containing kanamycin $(50 \mu \mathrm{g} / \mathrm{ml})$, and grown overnight at $37^{\circ} \mathrm{C}$. The overnight cultures were diluted $1: 100$ into fresh LB media containing $50 \mu \mathrm{g} / \mathrm{ml}$ kanamycin, and grown at $37^{\circ} \mathrm{C}$ with agitation until the optical density at $600 \mathrm{~nm}$ had reached between 0.6 and 0.8 . Protein expression was induced by adding $0.8 \mathrm{M}$ isopropyl-1-thio- $\beta$-D-galactopyranoside at a ratio of 1:1,500 for $3 \mathrm{~h}$. The cultures were centrifuged at $9,600 \mathrm{x} \mathrm{g}$ for $1 \mathrm{~min}$ at $4^{\circ} \mathrm{C}$, the supernatant was discarded and the cell pellets were dissolved in 5X SDS PAGE loading buffer. The expression level of the protein was analyzed using $10 \%$ SDS-PAGE, and the proteins were visualized using Commassie Brilliant Blue staining. The protein expression level of Ndufa10 was detected using western blotting.

Recombinant protein purification and measurement of the $M W$ of Ndufal0 variants. For the large-scale production of wild-type and mutant Ndufa10 proteins, the cell pellets were resuspended in $1 \mathrm{X}$ phosphate-buffered saline and sonicated on ice using an ultra-sonicator (x12,000; Misonix sonicator, Farmingdale, NY, USA) at $40 \%$ power, with 60 cycles 

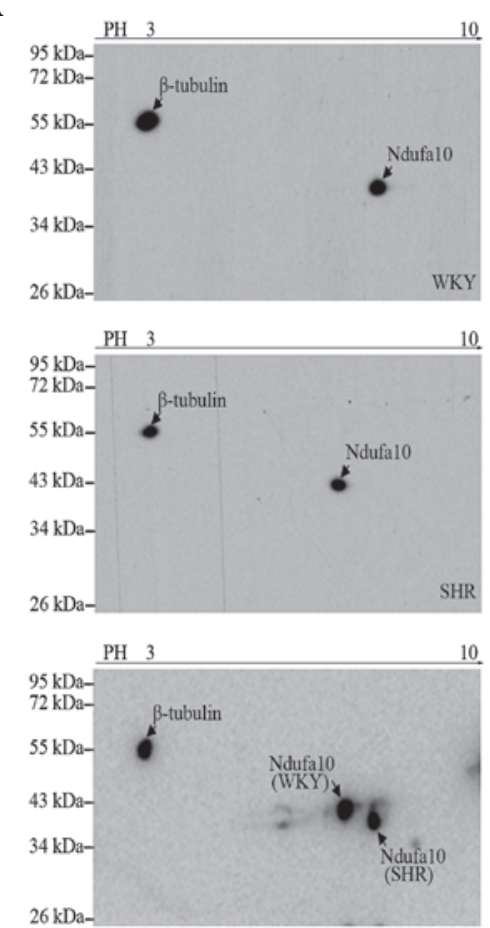
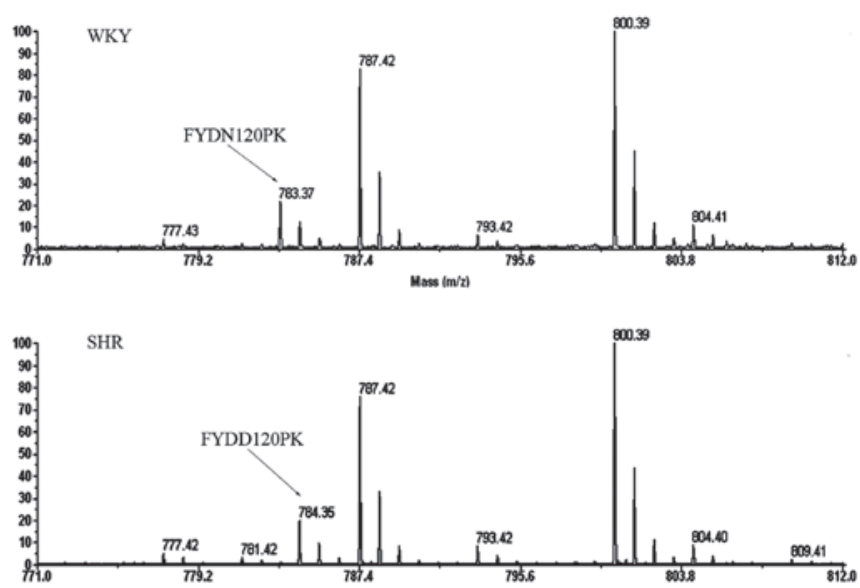

C

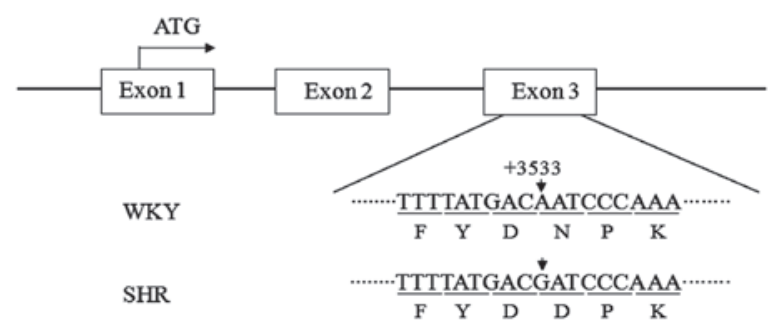

Figure 1. Ndufa10 variants in a 2D western blot and MALDI-TOF-TOF tandem MS. (A) A 2D-western blot of Ndufa10 in the left ventricle of WKY and SHR rats is shown. $\beta$-tubulin was used as an internal control. (B) The matched peptide, FYDNPK, obtained using MALDI-TOF-TOF tandem MS is shown in capital letters for the WKY protein, and the peptide, FYDDPK, of MALDI-TOF-TOF tandem MS shown in capital letters in for the SHR protein. The D/N substitution was at amino acid position 120 of the Ndufa10 amino acid sequence. (C) The expression of the Ndufa10 gene is shown, highlighting the genetic point mutation which is modulated in the SHR protein. MALDI-TOF-TOF tandem MS, matrix-assisted laser desorption/ionization-time of flight time-of-flight tandem mass spectrometry; Ndufa10, NADH dehydrogenase $1 \alpha$ subcomplex 10; SHR, spontaneously hypertensive rat; WKY, Wistar Kyoto.

of $5 \mathrm{sec}$ on/10 sec off. The suspensions were collected by centrifugation for $30 \mathrm{~min}$ at $20,000 \mathrm{x}$ g at $4^{\circ} \mathrm{C}$. The resulting pellets, containing inclusion bodies, were extracted twice in lysis buffer [10 mM imidazole, $100 \mathrm{mM} \mathrm{NaH} \mathrm{PO}_{4}$, $10 \mathrm{mM}$ Tris, $8 \mathrm{M}$ urea (pH 8.0)] for $30 \mathrm{~min}$ each at room temperature. The protein extracts were subsequently centrifuged for $30 \mathrm{~min}$ at $20,000 \mathrm{x}$ g at $4^{\circ} \mathrm{C}$. The supernatants were pooled, and equilibrated $\mathrm{Ni}^{2+}$-nitrilotriacetic acid (NTA) agarose was added (Qiagen, Manchester, UK) and placed on a rotary shaker at $4^{\circ} \mathrm{C}$ overnight. Following five washes with wash buffer [20 mM imidazole, $100 \mathrm{mM} \mathrm{NaH}{ }_{2} \mathrm{PO}_{4}, 10 \mathrm{mM}$ Tris and $8 \mathrm{M}$ urea $(\mathrm{pH} \mathrm{8.0)}$ ], the proteins were eluted with elution buffer [ $250 \mathrm{mM}$ imidazole, $100 \mathrm{mM} \mathrm{NaH} \mathrm{PO}_{4}, 10 \mathrm{mM}$ Tris and $8 \mathrm{M}$ urea $(\mathrm{pH} 8.0)]$ at $4^{\circ} \mathrm{C}$. The purity of the proteins was determined using 10\% SDS-PAGE with Coomassie Blue G-250 staining. The eluted fractions were desalted and concentrated using Centricon ${ }^{\circledR}$ YM-10 centrifugal filter devices (EMD Millipore) prior to the determination of the MW using matrix-assisted laser desorption/ionization-time of flight-mass spectrometry (MALDI-TOF-MS). Finally, the Ndufa10-120N and Ndufa10-120D proteins were mixed and diluted with rehydration buffer, prior to 2D electrophoresis.

Site-directed mutagenesis. A series of Ndufa10 mutations at amino acid position 120 were made using a PCR site-directed mutagenesis kit (Toyobo, Co., Ltd., Osaka, Japan) and pET28a-Ndufa10-120N as the template, according to the manufacturer's protocol. These mutations included neutral non-polar leucine (L), basic polar lysine $(\mathrm{K})$ and acidic polar glutamic acid (E) residues. All the mutants were confirmed using DNA sequencing. The production of recombinant Ndufa10 mutants was performed as described above. The primers used were as follows: 120E, forward: 5'-CTGTAG TTTAGAGAAATTTTATGACGAACCCAAAAGCAACGA CGGCAACAGCTA-3'; reverse: 5'-TAGCTGTTGCCGTCG TTGCTTTTGGGTTCGTCATAAAATTTCTCTAAACTAC AGC-3'; 120L, forward: 5'-GCTGTAGTTTAGAGAAATTTT ATGACCTTCCCAAAAGCAACGACGGCAACAGCTA-3'; reverse: 5'-TAGCTGTTGCCGTCGTTGCTTTTGGGAAGG TCATAAAATTTCTCTAAACTACAGC-3'; 120K, forward: 5'-GCTGTAGTTTAGAGAAATTTTATGACAAGCCCAAAA GCAACGACGGCAACAGCTA-3'; and reverse: 5'-TAGCTG TTGCCGTCGTTGCTTTTGGGCTTGTCAAAAATTTC TCTAAACTACAGC-3'.

\section{Results}

Detection of ndufa10 variants using $2 D$ western blot analysis and MALDI-TOF-TOF tandem MS. The Ndufa10 variant proteins from the cardiac tissue of SHR and WKY rats were detected using 2D western blot analysis, by which proteins were sequentially separated on the basis of their $\mathrm{pI}$ and size. As shown in Fig. 1A, the Ndufa10 protein from the SHR rats was identified to have lower $\mathrm{pI}$ value $(\sim 0.2 \mathrm{pH}$ unit difference) and a higher MW ( 2 $\mathrm{kDa}$ difference) compared with that from the WKY rats. Using MALDI-TOF-TOF tandem MS 


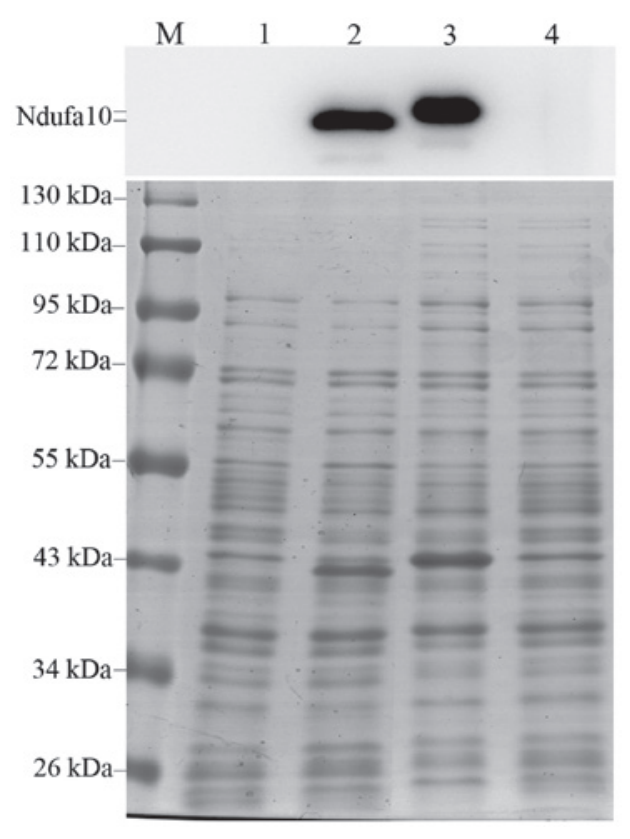

Figure 2. Detection of IPTG-induced expression of Ndufa10 protein in Escherichia coli BL21(DE3) cells. The pET28a-Ndufa10-120N and -120D plasmids were transformed into the BL21(DE3) strain of E. coli, and induced by the addition of $0.8 \mathrm{mM} \mathrm{IPTG}$ for $3 \mathrm{~h}$ at $37^{\circ} \mathrm{C}$. The protein expression levels were investigated using $10 \%$ SDS-PAGE with western blotting (upper panel) and Commassie Brilliant Blue staining (lower panel). Lane 1, Ndufa10-120N, uninduced; lane 2, Ndufa10-120N, induced with IPTG; lane 3, Ndufa10-120D, induced with IPTG; lane 4, Ndufa10-120D, uninduced; lane M, standard protein size markers $(\mathrm{kDa})$. IPTG, isopropyl-1-thio- $\beta$-D-galactopyranoside; Ndufa10, NADH dehydrogenase $1 \alpha$ subcomplex 10.

led to the detection of two different peptides, FYDN120PK and FYDD120PK, in samples from the WKY and SHR rats, respectively (Fig. 1B). The D/N substitution at amino acid position 120 resulting from an $\mathrm{A} / \mathrm{G}$ transition at position 358 in the coding region of Ndufa10 from the SHR rat was further confirmed by genomic DNA sequencing (RefGene NM_199495 range=chr9: 91655135-91689653; Fig. 1C).

Expression of the Ndufalo variants in vitro. In order to determine whether the apparent difference in MW between the two Ndufa10 variants was solely caused by the N-to-D substitution or post-translational modifications, initially, the MW of these two bacterially expressed recombinant Ndufa10 variants were compared. As shown in Fig. 2, the expression of both recombinant Ndufa10-120N and Ndufa10-120D Ndufa10 proteins was markedly induced, as revealed by Coomassie Brilliant Blue staining (the bottom panel) and western blotting (the top panel). Similarly to what was previously observed with rat tissue samples in our laboratory, the apparent $\mathrm{MW}$ of the Ndufa10-120D protein was $\sim 2 \mathrm{kDa}$ higher compared with that of Ndufa10-120N, suggesting that the N-to-D substitution was sufficient to cause the shift in its MW.

Purification and $M W$ measurement of the Ndufalo variants. To measure the MW more precisely, bacterially expressed, histidine-tagged recombinant Ndufa10 proteins from the two groups were assessed using $\mathrm{Ni}^{2+}-\mathrm{NTA}$ agarose gel electrophoresis under denaturing conditions. The purification processes were revealed to yield high-purity proteins for both
A

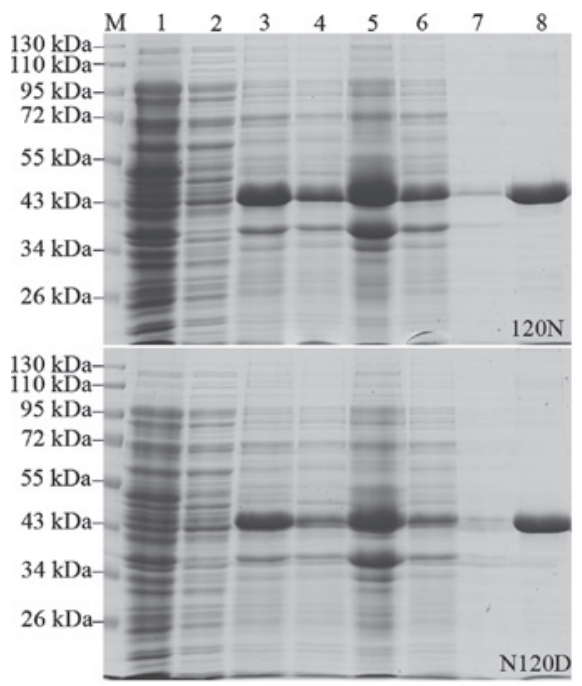

B

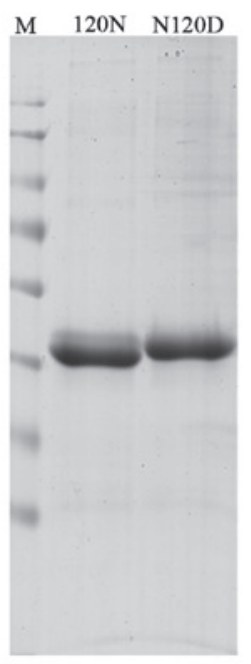

C
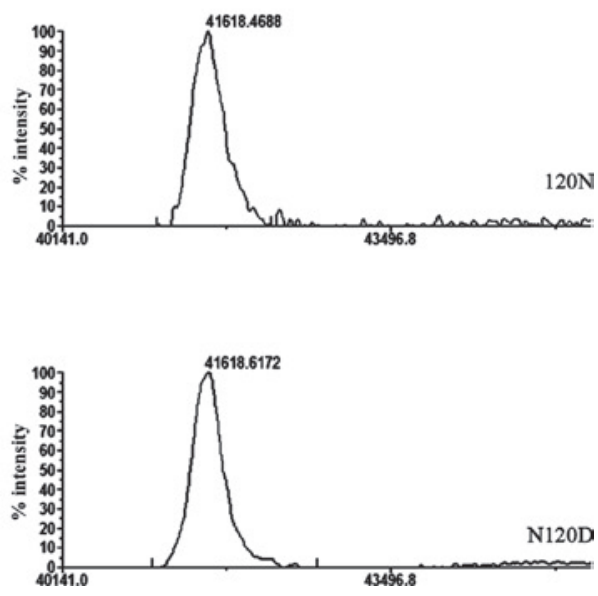

Figure 3. Purification of the Ndufa10 fusion proteins, and the practical determination of the MW of the Ndufa $10-120 \mathrm{~N}$ and $-120 \mathrm{D}$ proteins. (A) Protein purification of the Ndufa10-120N (upper panel) and the Ndufa10-120D (lower panel) proteins is shown. Lane 1, uninduced cells; lane 2, supernatant of the IPTG-induced cells; lane 3, the IPTG-induced cells after a duration of $3 \mathrm{~h}$; lane 4 , cell lysate; lane 5 , precipitate following centrifugation; lane 6 , supernatant of $\mathrm{Ni}^{2+}$-NTA agarose combined protein; lane 7, wash with lysis buffer; lane $8,250 \mathrm{mM}$ imidazole elution; lane $\mathrm{M}$, standard protein size markers (kDa). (B) Separation of the proteins using 10\% SDS-PAGE, and detection of the protein bands by Commassie Brilliant Blue staining (C) Matrix-assisted laser desorption/ionization-time of flight/time-of-flight tandem mass spectrometric analysis of the purified recombinant Ndufa10 protein variants. IPTG, isopropyl-1-thio- $\beta$-D-galactopyranoside; Ndufa10, NADH dehydrogenase $1 \alpha$ subcomplex 10; NTA, nitrilotriacetic acid. 


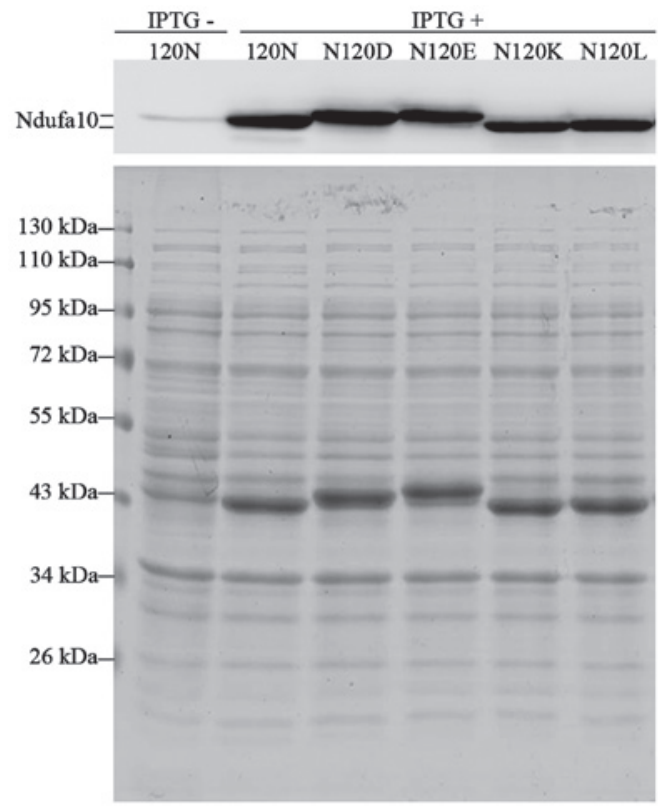

Figure 4. Expression of recombinant Ndufa10 variants at position 120 in E. coli BL21(DE3) cells. The relative migrations of Ndufa10-120D and $-120 \mathrm{E}$ were both markedly slower than that of other Ndufa10 variants. Cell lysates were subjected to SDS-PAGE separation stained with Coomassie Brilliant Blue (lower panel), and the upper panel shows a representative western blot for the detection of the protein expression of Ndufa10. IPTG, isopropyl-1-thio - $\beta$-D-galactopyranoside; Ndufa10, NADH dehydrogenase $1 \alpha$ subcomplex 10.

Ndufa10-120N and Ndufa10-120D (Fig. 3A). Purified, recombinant Ndufa10-120N and Ndufa10-120D proteins, and a mixture of equal quantities of them, were subjected to $2 \mathrm{D}$ electrophoretic separation. As shown in Fig. 3B, the recombinant Ndufa10-120N and Ndufa10-120D proteins appeared as two distinct bands, although a shift in the MW was observed with the Ndufa10-120D protein, similarly to what was observed previously in our laboratory with SHR rats. Further analysis using MALDI-TOF-TOF tandem MS revealed an experimental MW for the Ndufa10-120N protein of 41,618.4688 Da (theoretical MW, 41,655.49 Da), whereas the experimental MW of the Ndufa10-120D protein was 41,618.6172 Da (theoretical MW, 41,656.47 Da), further confirming that the difference in mobility in SDS-PAGE was caused by the N/D substitution (Fig. 3C).

Effect of acidic amino acid substitutions on the apparent MW of the Ndufal0 protein. To further explore whether the reported phenomena were attributable solely to the N120D substitution, the influence of different amino acids at the position 120 on the mobility of the Ndufa10 proteins on SDS-PAGE was subsequently investigated. To this end, several Ndufa10 mutant proteins were generated at residue position 120 by replacing non-polar Asn (N; MW, 114.11 Da) with neutral, non-polar Leu (L; MW, 113.16 Da), with basic, polar Lys (K; MW, 128.17 Da), or with acidic, polar Glu (E; MW, $129.12 \mathrm{Da})$, in addition to Asp (D; MW, 115.09 Da). As shown in Fig. 4, the Ndufa10-120D protein demonstrated a similar shift, as described above. The Ndufa10-120K and Ndufa10-120L proteins exhibited the same mobility as Ndufa10-120N, whereas the Ndufa10-120E mutant revealed the highest $\mathrm{MW}$ of all. Even after having taken into consideration the MW of the amino acids, the negative charge of the amino acid in position 120 is therefore revealed to be the major factor accounting for the mobility of the protein in the SDS-PAGE gel.

\section{Discussion}

The predominant findings of the present study were: (i) that WKY and SHY rats express different variants of the mitochondria complex I subunit Ndufa10 protein, Ndufa10-120N and Ndufa10-120D, respectively; (ii) that the Ndufa10-120D protein is shifted to a higher MW and a lower pI compared with the Ndufa10-120N protein; (iii) that Ndufa10-120D is specifically expressed in the SHR rats, whereas the WKY rats express Ndufa10-120N; and (iv) that the in-gel MW shift of the Ndufa10-120D protein is not caused by additional post-translational modifications, since an in-gel mobility difference exists between bacterially expressed recombinant Ndufa10-120N and Ndufa10-120D proteins. However, the two recombinant variants exhibited an almost identical experimental MW, as determined by the MALDI-TOF MS analysis.

The in-gel mobility is influenced by various properties of the protein. Among them, hydrophobicity has the greatest effect. Statistical analysis revealed that hydrophilic proteins tend to migrate more slowly (12). In general, SDS binds to a protein at a ratio of approximately one molecule/two amino acid residues. However, because this binding is mediated via hydrophobic interactions, SDS preferentially binds to the hydrophobic region of proteins. As a result, it is likely that hydrophobic proteins bind larger quantities of SDS than hydrophilic ones, and therefore migrate faster on SDS-PAGE analysis. Furthermore, the pI values of proteins also influence gel mobility to a certain extent. Acidic proteins exhibited a lower mobility than predicted, which may be due to the presence of a negative charge repulsion effect with SDS (12). Negatively charged, acidic aspartic acid residues are more hydrophilic than uncharged, polar asparagines, and therefore the Ndufa10-120D protein exhibited a lower $\mathrm{pI}$, and slower migration, compared with the Ndufa10-120N protein. Furthermore, the Ndufa-120K or the Ndufa-120L mutated proteins migrated very similarly to the Ndufa10-120N protein due to their hydrophobic properties. It is notable that the Ndufa-120E mutant migrated differently, although the difference was comparable with that exhibited by Ndufa10-120D, since the negatively charged, acidic glutamic acid residue also has hydrophilic properties.

Unlike the other complex I components, which are mostly hydrophobic, the Ndufa10 subunit is relatively hydrophilic, which suggests that it interacts less strongly with other components of complex I and it is more accessible to external interactions with other non-complex I proteins, including cAMP-dependent protein kinase or phosphatase $(8,10,13)$. Thus far, many mutations in complex I subunits have been associated with human neurodegenerative diseases (14). Mutations in exons 1 and 3 of the Ndufa10 gene were previously found to cause complex I deficiency in a patient with Leigh disease (15). Ndufa10 has a phylogenetically conserved GAT codon, coding for Asp at position 120. A previous report demonstrated that animals homozygous for AAT or GAT only exhibited the Ndufa10-120N or Ndufa10-120D isoforms, respectively, whereas the presence of the two alleles was associated with the detection of both variants using SDS-PAGE 
and 2D gel electrophoresis (7). All the animals (normal and SHY rats) used in the present study were homozygous for A/A or $\mathrm{G} / \mathrm{G}$ in both alleles, respectively. It appears that the presence of the $\mathrm{G} / \mathrm{G}$ genotype leads to a greater susceptibility for rats to develop hypertension. Whether A/G heterozygous rats were susceptible to developing hypertension was not known during the course of the present study, since heterozygous rats were not obtained from the Shanghai SLAC Laboratory Animal Co., Ltd. Therefore, it would be interesting to know whether the N/D substitution influences the function of Ndufa10 and complex I, and, consequently, is associated with the pathophysiology of spontaneous hypertension in rats. Most importantly, it remains to be elucidated whether such an amino acid replacement occurs in humans. Further studies are required to demonstrate whether the Ndufa10 DNA polymorphism at position 358 is a disease-causing mutation, and to determine its potential role as a biomarker to predict the risk of developing hypertension.

\section{Acknowledgements}

This study was supported by a grant from the Shanghai Natural Science Foundation (grant no. 11ZR1421500), and by sponsorship received from a Shanghai Pujiang Programa grant (grant no. 14PJ1406300). This study was also supported in part by grants from the Ministry of Science and Technology of China (grant no. 2013CB910903).

\section{References}

1. Di Lisa F, Canton M, Menabò R, Kaludercic N and Bernardi P. Mitochondria and cardioprotection. Heart Fail Rev 12: 249-260, 2007.

2. Lopez-Campistrous A,HaoL, Xiang W, Ton D, Semchuk P, Sander J, Ellison MJ and Fernandez-Patron C: Mitochondrial dysfunction in the hypertensive rat brain: Respiratory complexes exhibit assembly defects in hypertension. Hypertension 51: 412-419, 2008.

3. Takimoto E and Kass DA: Role of oxidative stress in cardiac hypertrophy and remodeling. Hypertension 49: 241-248, 2007.
4. Bernal-Mizrachi C, Gates AC, Weng S, Imamura T, Knutsen RH, DeSantis P, Coleman T, Townsend RR, Muglia LJ and Semenkovich CF: Vascular respiratory uncoupling increases blood pressure and atherosclerosis. Nature 435: 502-506, 2005.

5. Smeitink JA, van den Heuvel LW, Koopman WJ, Nijtmans LG, Ugalde $\mathrm{C}$ and Willems PH: Cell biological consequences of mitochondrial NADH: Ubiquinone oxidoreductase deficiency. Curr Neurovasc Res 1: 29-40, 2004.

6. Efremov RG and Sazanov LA: Structure of the membrane domain of respiratory complex I. Nature 476: 414-420, 2011.

7. Meng C, Jin X, Xia L, Shen SM, Wang XL, Cai J, Chen GQ, Wang LS and Fang NY: Alterations of mitochondrial enzymes contribute to cardiac hypertrophy before hypertension development in spontaneously hypertensive rats. J Proteome Res 8: 2463-2475, 2009.

8. Sardanelli AM, Technikova-Dobrova Z, Scacco SC, Speranza F and Papa S: Characterization of proteins phosphorylated by the cAMP-dependent protein kinase of bovine heart mitochondria. FEBS Lett 377: 470-474, 1995.

9. Schulenberg B, Aggeler R, Beechem JM, Capaldi RA and Patton WF: Analysis of steady-state protein phosphorylation in mitochondria using a novel fluorescent phosphosensor dye. J Biol Chem 278: 27251-27255, 2003.

10. Schilling B, Aggeler R, Schulenberg B, Murray J, Row RH, Capaldi RA and Gibson BW: Mass spectrometric identification of a novel phosphorylation site in subunit NDUFA10 of bovine mitochondrial complex I. FEBS Lett 579: 2485-2490, 2005.

11. Muñoz J, Fernández-Irigoyen J, Santamaría E, Parbel A, Obeso J and Corrales FJ: Mass spectrometric characterization of mitochondrial complex I NDUFA10 variants. Proteomics 8: 1898-1908, 2008

12. Shirai A, Matsuyama A, Yashiroda Y, Hashimoto A Kawamura Y, Arai R, Komatsu Y, Horinouchi S and Yoshida M: Global analysis of gel mobility of proteins and its use in target identification. J Biol Chem 283: 10745-10752, 2008.

13. Lazarou M, McKenzie M, Ohtake A, Thorburn DR and Ryan MT: Analysis of the assembly profiles for mitochondrialand nuclear-DNA-encoded subunits into complex I. Mol Cell Biol 27: 4228-4237, 2007.

14. Distelmaier F, Koopman WJ, van den Heuvel LP, Rodenburg RJ, Mayatepek E, Willems PH and Smeitink JA: Mitochondrial complex I deficiency: From organelle dysfunction to clinical disease. Brain 132: 833-842, 2009.

15. Hoefs SJ, van Spronsen FJ, Lenssen EW, Nijtmans LG, Rodenburg RJ, Smeitink JA and van den Heuvel LP: NDUFA10 mutations cause complex I deficiency in a patient with Leigh disease. Eur J Hum Genet 19: 270-274, 2011. 